\title{
Divertículo duodenal intraluminal como causa de obstrucción duodenal
}

\section{Intraluminal duodenal diverticulum as a cause of duodenal obstruction}

\author{
Rocio Pérez-Quintero' $\mathbb{D}$, Marcos Alba-Valmorisco ${ }^{2} \mathbb{D}$, Juan Candón-Vázquez ${ }^{2} \mathbb{D}$, \\ Daniel Bejarano González-Serna² $\mathbb{D}$, Rafael Balongo-García ${ }^{3}$ D
}

1 Médica, especialista en Cirugía general, Unidad de esofagogástrica y endocrino, Servicio de Cirugía General y del Aparato digestivo, Hospital Juan Ramón Jiménez, Huelva, España.

2 Médico, especialista en Cirugía general, Unidad Hepatobiliopancreática, Servicio de Cirugía General y del Aparato digestivo, Hospital Juan Ramón Jiménez, Huelva, España.

3 Médico, especialista en Cirugía general, Unidad de esofagogástrica; jefe, Servicio de Cirugía General y del Aparato digestivo, Hospital Juan Ramón Jiménez, Huelva, España.

\section{Resumen}

Introducción. El divertículo duodenal intraluminal, también conocido como windsock diverticulum, es una causa rara de dolor abdominal intermitente y plenitud postpandrial, que puede complicarse con obstrucción, sangrado, pancreatitis o colangitis. Suele cursar de forma asintomática y ante la aparición de síntomas el tratamiento de elección es quirúrgico.

Caso clínico. Presentamos el caso de una mujer de 24 años, sin antecedentes de interés, que es estudiada por dolor abdominal y plenitud postpandrial, que resulta finalmente en una obstrucción intestinal alta. Tras estudio exhaustivo y necesidad de una cirugía previa, es diagnosticada de un divertículo duodenal intraluminal.

Conclusión. La paciente se trató mediante cirugía con resolución exitosa del cuadro de obstrucción intestinal.

Palabras clave: duodeno; enfermedades duodenales; divertículo; obstrucción duodenal; obstrucción intestinal; cirugía.

\begin{abstract}
Introduction: Intraluminal duodenal diverticulum, also known as a windsock diverticulum, is a rare cause of intermittent abdominal pain and postprandial fullness, which can be complicated by obstruction, bleeding, pancreatitis, or cholangitis. It is usually asymptomatic and when symptoms appear, the treatment of choice is surgical.

Clinical case: We present the case of a 24-year-old woman with no relevant history who is studied for abdominal pain and postprandial fullness, presented with an upper intestinal obstruction. After an exhaustive study and the need for a previous surgery, she was diagnosed with an intraluminal duodenal diverticulum.

Conclusion: The patient was treated by surgery with successful resolution of the intestinal obstruction.

Keywords: duodenum; duodenal diseases; diverticulum; duodenal obstruction; intestinal obstruction; surgery.

Fecha de recibido: 19/01/2021 - Fecha de aceptación: 01/03/2021 - Publicación en línea: 06/10/2021

Correspondencia: Rocío Pérez-Quintero, Calle Antonio Rodriguez Waflar, no13, Beas, Huelva, España, 21630. Teléfono: +34635565956. Direccion electrónica: roc14589@hotmail.com

Citar como: Pérez-Quintero R, Alba-Valmorisco M, Candón-Vázquez J, González-Serna DB, Balongo-García R. Divertículo duodenal intraluminal como causa de obstrucción duodenal. Rev Colomb Cir. 2022;37:142-5. https://doi.org/10.30944/20117582.865

Este es un artículo de acceso abierto bajo una Licencia Creative Commons - BY-NC-ND https://creativecommons.org/licenses/by-nc-nd/4.0/deed.es
\end{abstract}




\section{Introducción}

El divertículo duodenal intraluminal (DDI), también conocido como windsock diverticulum, es una causa rara de dolor abdominal intermitente y plenitud postpandrial, que puede complicarse con obstrucción, sangrado, pancreatitis o colangitis. La frecuencia reportada es de hasta $22 \%$ en autopsias, siendo sintomáticos sólo un $10 \%$ y requiriendo tratamiento el $1 \%{ }^{1}$.

Se trata de una anormalidad congénita, que parece ser el resultado de una recanalización incompleta de la luz del intestino anterior embriológico, sobre la octava semana de gestación ${ }^{2}$. En su desarrollo, inicialmente hay hiperplasia de las células epiteliales de la mucosa duodenal y, como resultado de años de fuerzas peristálticas y falta de inervación, hay una distensión progresiva del tejido para formar un divertículo de tipo pulsional. El lugar más frecuente de formación es la segunda porción duodenal, justo distal a la ampolla de Vater ${ }^{3}$.

Puede estar asociado a otras entidades como páncreas anular, variaciones en la ubicación y estructura de los conductos biliares y pancreáticos, coledococele, malrotación intestinal, e incluso situs inversus.

\section{Caso clínico}

Presentamos el caso de una mujer de 24 años, sin antecedentes personales de interés, con cuadro clínico de plenitud postpandrial y dolor en epigastrio de un año de evolución, con empeoramiento en los últimos meses y pérdida de peso, que precisa ingreso hospitalario para estudio exhaustivo. A la exploración presentaba dolor en epigastrio, leve, con ligera distensión y timpanismo.

Se realizó una endoscopia de vías digestivas altas (EVDA) y una ecografía, sin encontrar hallazgos de interés, por lo que posteriormente se solicitó una radiografía de tránsito gastrointestinal, donde se visualizó una imagen de invaginación duodenal a nivel de la segunda rodilla, con dilatación anterógrada e incremento de peristalsis. Para completar el estudio se realizaron una Tomografía computarizada (TC) con contraste intravenoso y una ecoendoscopia (USE), que corroboraron los hallazgos del tránsito.

Se decidió tratamiento quirúrgico programado, mediante laparotomía a través de una incisión subcostal bilateral, visualizándose un megaduodeno a expensas de segunda y tercera porción, sin evidenciar invaginación u otra causa, por lo que se decidió realizar una pexia del duodeno tras maniobra de Cattell y Kocher.

Tras dicha cirugía, la paciente no evoluciona favorablemente, presentando un cuadro obstructivo con náuseas y alto débito por la sonda nasogástrica (SNG), con empeoramiento de parámetros nutricionales. Se realizó un nuevo tránsito gastrointestinal y EVDA (Figura 1), donde se observa un divertículo duodenal intraluminal gigante a

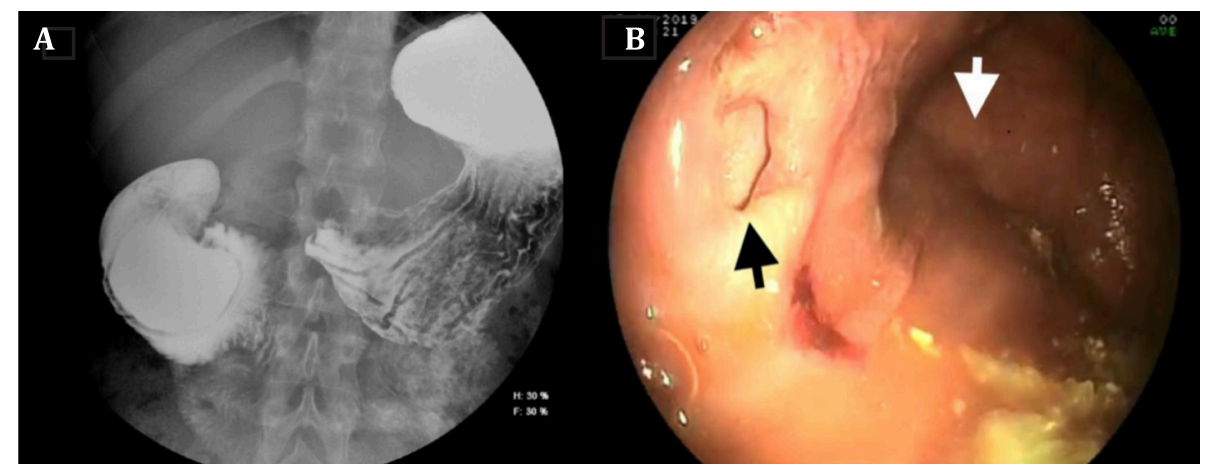

Figura 1. A) Segundo estudio de tránsito gastrointestinal con bario, donde se observa un divertículo duodenal intraluminal gigante, que condiciona oclusión duodenal con dilatación del duodeno proximal. B) En la endoscopia, la flecha blanca marca el saco del divertículo y la flecha negra, la verdadera luz duodenal. 
nivel de la segunda porción, que condiciona una oclusión duodenal con dilatación del duodeno proximal. Ante los hallazgos, se decide nuevamente tratamiento quirúrgico programado. Durante la segunda cirugía, se realizó una duodenotomía en segunda porción duodenal, identificando un gran divertículo cercano a la papila. Se practicó diverticulectomía, colocando una sonda como tutor en la papila a través del conducto cístico, para proceder al cierre de la duodenotomía (Figura 2).

La paciente presentó una evolución postoperatoria sin incidencias y fue dada de alta a los 9 días. La revisión en consulta ambulatoria al mes cursa sin alteraciones, con buena tolerancia a la dieta y con un adecuado perfil nutricional. Los resultados de anatomía patologica no mostraron malignidad, solo hiperplasia mucosa.

\section{Discusión}

En general, los DDI cursan de forma asintomáti$\mathrm{ca}^{4}$, pero cuando aparecen los síntomas, suelen

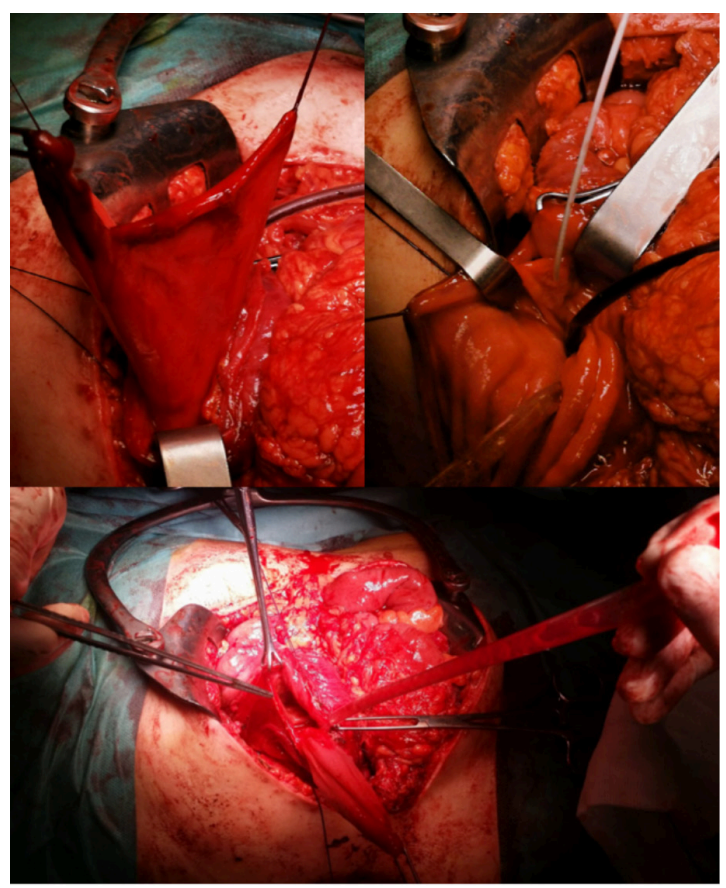

Figura 2. Tras realizar duodenotomía se observa un gran divertículo cercano a la papila, que precisó la inserción de una sonda como tutor previo a la realización de diverticulectomía. darse entre la tercera y la cuarta décadas de la vida. Se trata de síntomas inespecíficos como dolor epigástrico, plenitud postpandrial, náuseas o vómitos. Debido a esto, pueden experimentar pérdida de peso e incluso desnutrición ${ }^{4}$. Las complicaciones del divertículo son menos frecuentes e incluyen la hemorragia gastrointestinal ${ }^{3}$, pancreatitis ${ }^{5}$, colangitis u obstrucción duodenal, que con frecuencia es intermitente.

El diagnóstico es incidental en la mayoría de los casos. Una gran parte son diagnosticados mediante estudio imaginológico contrastado de tránsito gastrointestinal, seguido de endoscopia de vías digestivas altas (EVDA) y, con menor frecuencia, de TC. La apariencia endoscópica es un desafío debido a que el DDI puede colapsarse y pasar desapercibido. Además, puede ser confuso si el endoscopista no está familiarizado con esta rara entidad. La videocápsula endoscópica tiene un éxito de 38 a 83 \% para el diagnóstico de divertículos yeyunales. La arteriografía mesentérica o la gammagrafía con enterocitos marcados pueden usarse ante casos de hemorragia activa ${ }^{6}$.

Entre los diagnósticos diferenciales se encuentran coledococele distal, masa quística ampular y quiste de duplicación duodenal.

El tratamiento está indicado en los casos sintomáticos. La intervención quirúrgica es el tratamiento de elección, ya que el tratamiento conservador tiene alta tasa de recurrencia. Sin embargo, las nuevas técnicas endoscópicas permiten abordar esta patología con buenos resultados y con los beneficios que supone evitar la opción quirúrgica en el paciente ${ }^{2}$. Se han descrito varias técnicas endoscópicas como la diverticulectomía o la diverticulotomía. No obstante, aún hay escasez de resultados publicados a largo plazo para poder comparar las técnicas endoscópicas con la cirugía $^{7,8}$. En nuestro caso no tuvimos la posibilidad de tratamiento endoscopio por la falta de disponibilidad en nuestro centro.

Algunos estudios recomiendan la escisión laparoscópica debido a la posibilidad de explorar la cavidad abdominal en busca de otras anormalidades anatómicas intestinales significativas y la capacidad de facilitar la escisión completa del 
diverticulo mientras se mantiene la integridad de los conductos biliar y pancreático 9 .

Según la literatura, los procedimientos quirúrgicos realizados, por orden de frecuencia, son la escisión completa del divertículo, gastroyeyunostomía, duodenoyeyunostomia o esfinteroplastia, entre otros ${ }^{8}$, pero sigue siendo el procedimiento de elección la duodenotomía y escisión del divertículo. Se recomienda la identificación de la ampolla de Vater para preservar el drenaje biliar y pancreático, incluso antes de la escisión del divertículo, puesto que estos pueden desembocar en él. Es recomendable la realización de una colangiografía/pancreatografia intraoperatoria para asegurar ambos drenajes.

\section{Conclusión}

Tener presente la sospecha de esta rara entidad es fundamental para un diagnóstico temprano y un tratamiento adecuado. La EVDA y el tránsito gastrointestinal son las pruebas más útiles para confirmar el diagnóstico. La cirugía es el tratamiento de elección en la actualidad, sin embargo, el avance en las técnicas endoscópicas abre una posibilidad terapéutica en casos seleccionados.

\section{Cumplimientos de normas éticas}

Consentimiento informado: Para la publicación de este caso, se obtuvo el consentimiento informado por parte de la paciente.

Conflictos de interés: Los autores declararon no tener conflictos de interés en la elaboración del presente artículo.

Financiación: Autofinanciado por los autores.

\section{Contribución de los autores}

Concepción y diseño del estudio: Rocío Pérez- Quintero

Adquisición de datos: Marcos Alba-Valmorisco, Juan Candón-Vázquez

Análisis e interpretación de datos: Daniel Bejarano González-Serna, Rocío Pérez-Quintero
Redacción del manuscrito: Rocío Pérez-Quintero

Revisión crítica: Rafael Balongo-García, Marcos AlbaValmorisco, Juan Candón-Vázquez, Daniel Bejarano González-Serna

\section{Referencias}

1. Saavedra VL, Kane TD, Garrity SH. Surgical management of symptomatic intraluminal duodenal diverticulum in an adolescent. Glob Pediatr Health. 2018;5:2333794X18755240. https://doi.org/10.1177/2333794X18755240

2. Law R, Topazian M, Baron TH. Endoscopic treatment of intraluminal duodenal ("windsock") diverticulum: varying techniques from five cases. Endoscopy. 2012;44:1161-4. https://doi.org/10.1055/s-0032-1325757

3. Mahajan SK, Kashyap R, Chandel UK, Mokta J, Minhas SS. Duodenal diverticulum: Review of literature. Indian J Surg. 2004;66:140-5.

4. Reichert MC. Recurrent pancreatitis caused by a huge intraluminal duodenal diverticulum. J Gastrointest Liver Disease. 2012;21:126.

5. Clemente G, Sarno G, Giordano M, De Rose AM, Nuzzo G. Intramural duodenal diverticulum mimicking a periampullary neoplasm. Am J Surg. 2008;196:31-2. https://doi.org/10.1016/j.amjsurg.2007.10.028

6. Villarreal RA, Vinck EE, Cabrera LF, Zuchini AC, JiménezC. Divertículo único y verdadero de yeyuno con obstrucción intestinal y abdomen agudo. Rev Colomb Cir. 2019;34:69-4.

https://doi.org/10.30944/20117582.100

7. Anand V, Provost J, Bakr M, Bach C, Merchant P, Brown $\mathrm{C}$, Gruss $\mathrm{C}$. Two cases of intraluminal "windsock" diverticula resulting in partial duodenal obstruction. ACG Case Rep J. 2016;3:135. https://doi.org/10.14309/crj.2016.108

8. D’Alessio MJ, Rana A, Martin JA, Moser J. Surgical management of intraluminal duodenal diverticulum and coexisting anomalies. J Am Coll Surg. 2005;201:143-8. https://doi.org/10.1016/j.jamcollsurg.2005.03.033

9. Meinke AK, Meighan DM, Meinke ME, Mirza N, Parris TM, Meinke RK. Intraluminal duodenal diverticula: Collective review with report of a laparoscopic excision. Journal of laparoendoscopic \& advanced surgical techniques. 2013; 23:129-36.

https://doi.org/10.1089/lap.2012.0236 